\title{
Neutralización ácido - base, un concepto desde lo cotidiano
}

\author{
Acid base neutralization, a daily basis concept \\ Juan Ricardo Muños y Liz Muñoz
}

Universidad Distrital Francisco José de Caldas, Bogotá-Colombia. richard11627@hotmail.com, lizm200528@yahoo.com

\section{Resumen}

A continuación presentamos una experiencia de aula realizada por los docentes en formación de la Universidad Distrital, sobre la enseñanza del concepto de neutralización ácido - base, esto con la finalidad de generar una propuesta innovadora en cuanto a las metodologías y modelos utilizados para la enseñanza de las ciencias en el aula. La propuesta se fundamenta en el modelo de resolución de problemas y la contextualización de la ciencia desde lo cotidiano.

\section{Palabras clave}

Neutralización, ácido - base, innovación, propuesta de aula, resolución de problemas.

\section{Abstract}

Next we presented a dassroom experience performed by the formation teachers of the Universidad Distrital, about the teaching concept of the acid - base neutralization, this is in order to generate an innovating proposal as far as the methodologies and models used for the teaching of sciences in the classroom. The proposal is based on the model of resolution of problems and the contextualisation of science on a daily basis.

\section{Keywords}

Neutralization, acid - bases, innovation, proposal of dassroom, resolution of problems

\section{Introducción}

El desarrollo de las ciencias, a través de los tiempos ha involucrado dos dases de lenguaje, uno científico y otro cotidiano. Por lo general la comunidad científica ha desarrollado una terminología propia que solo es utilizada por ellos. Por otro lado la ciencia también hace uso de términos que son muy utilizados tanto en el laboratorio como en la vida cotidiana, es decir, tienen gran aceptación tanto en el ámbito cotidiano como en el cient́fico.

En el primer caso, el aprendizaje de los conceptos, se da en situaciones determinadas y su uso se realiza únicamente en el ámbito cient́fico. En el segundo caso, alguno de los conceptos que se tratan de enseñar al estudiante han sido trabajados al interior del aula, por lo que se dice que se poseen algunas ideas previas sobre estos, lo cual puede llegar a generar conceptos que se alejan de los contenidos cient́ficos que se le enseñan al estudiante. Un ejemplo de esta problemática se puede evidenciar, cuando se utiliza el término neutralización y el estudiante solo, lo asocia a la existencia de ácidos fuertes y bases fuertes únicamente y su carácter neutro al reaccionar estos dos compuestos, pero no se tienen en cuenta otras características que pueden intervenir en el proceso, que no sólo tienen que ver con alcanzar un punto de equivalencia asociado por lo general con pH. 
Este trabajo tenía como objetivo, diseñar una propuesta didáctica para la enseñanza del concepto de neutralización ácido-base, a partir de los conceptos previos del estudiante, evidenciando cuales son las principales dificultades que tienen los estudiantes para comprender el concepto y así crear estrategias que faciliten su aprendizaje, encaminado esto a su diario vivir para que el estudiante pueda tener una asociación de dichos conceptos con su vida y así se genere tanto un cambio conceptual como un aprendizaje significativo.

\section{La conceptualización sobre los ácidos}

Para elaborar una propuesta de enseñanza sobre los conceptos seleccionados, se hizo necesario hacer una revisión desde lo histórico con el fin de contextualizar la propuesta, de este modo se pudo hacer el análisis de que el desarrollo histórico de los conceptos de ácidos y bases está ligado al descubrimiento y la utilización a través de la historia de estos conceptos, así como de la dasificación de estas sustancias a partir de fenómenos observables. De este modo se enfatizó en que no se puede estudiar estos conceptos sin contextualizar lo que se entendía por ácido o por base en cada momento histórico.

Los productos que ahora consideramos como ácidos y como bases se conocen desde la antigüedad, como lo ponen de manifiesto algunas recetas prácticas encontradas en papiros de la cultura helenística (en Estocolmo y en Leiden). A pesar de que no se haća ninguna interpretación de lo que ocurría en los procesos que se describen, en estas recetas sí se utilizaban el vinagre y los zumos de frutas como disolventes de ciertos metales, y se conocían algunas sales como la sal común, el carbonato de sodio, el sulfato de hierro, entre otras. Así mismo, durante la edad media, concretamente en el mundo árabe, también se utilizaron recetas de tipo práctico como, por ejemplo, la de al-Raz, en la que aparece la primera dasificación en la cual se engloban varias sustancias atendiendo a cualidades semejantes.

A lo largo del siglo XIII, en el Occidente cristiano, como consecuencia del perfeccionamiento en las técnicas alquimistas, concretamente de la destilación, se obtuvieron los ácidos minerales. Con su descubrimiento, los químicos pudieron disolver más sustancias y realizar más reacciones en disolución, que con los ácidos orgánicos que hasta entonces se utilizaban. Pero, a pesar de que se perfeccionaron las técnicas, se utilizaron un mayor número de ácidos minerales y con ellos se aumentaron los procesos en los que participaban, por lo que no llegó a interpretarse teóricamente qué ocurría en esos procesos ni la naturaleza de las sustancias descubiertas.

Las reflexiones realizadas para entonces sobre la naturaleza de los ácidos, las bases y las sales representaron un intenso debate que se estableció en este siglo, que enfrentaba a los que afirmaban que el ácido era el agente único o universal, frente a los que defendían el antagonismo entre ácido y álcali. Entre éstos últimos Boyle, definía los ácidos por su efervescencia en contacto con los álcalis o viceversa. El debate se resolvió a favor de éstos al demostrar Boyle que, los ácidos podían descomponerse y que un gran número de auerpos no contenían ningún ácido, por lo que no podía constituir un agente universal. A este autor se debe la primera dasificación de ácidos y bases basada en fenómenos observables (ser corrosivos, disolver metales, reaccionar con ácidos o con bases, el color de las disoluciones ácidas o básicas con indicadores como el licor de violetas, etc.).

Como se ha indicado en párrafos anteriores, durante siglos se produjo una acumulación preteórica de conocimientos químicos (utilización de ácidos, dasificación de las sustancias ácidas y básicas, perfeccionamiento de los trabajos prácticos, etc.). Ello condujo a que en el siglo XVIII se utilizara la neutralización como la reacción entre un ácido y una base para formar una sal (estos tres términos definidos según sus propiedades observables descritas por Boyle). La utilización de este término se aplicó tanto a las soluciones acuosas como en la fabricación de vidrios donde reaccionaban tierras ácidas con otras básicas a altas temperaturas. 
Con la introducción de un auerpo teórico de conocimientos (la química del siglo XIX), se buscó explicación a estos procesos. Por ejemplo, desde la teoría de la disociación iónica de Arnhenius (1887), en la que relacionó las propiedades de los ácidos con la presencia de iones hidrógeno libres y las de las bases con los iones hidróxido; un producto neutro sería aquél que no cediera iones hidrógeno ni hidróxido. En esta teoría se establece una escala absoluta de dasificación de las sustancias como ácidas, neutras o básicas según la constante de su disociación electrolítica.

La forma de presentación clásica de las teorías ácido-base a los estudiantes universitarios y de secundaria consiste en exponer la definición de lo que cada una entiende por ácido, por basey por neutralización, este último concepto entendido como proceso que transcurre auando se ponen en contacto los ácidos y las bases: según las teorías inotrópicas o teoría de los disolventes, inspirada en los postulados de Franklin (1912), las sustancias se dasifican como «cationotrópicas»o «anionotrópicas 》 según el ion que se transfiera. Así en un sistema anionotrópico, los ácidos se definen como «aceptadores 》 de aniones y las bases como «dadoras» de aniones y, por el contrario, en un sistema cationotrópico las bases son las que aceptan los cationes y los ácidos los que los ceden. Por lo que la neutralización, según la teoría de los disolventes, es la combinación del anión característico del disolvente con el catión característico del disolvente para formar éste.

En 1923, Brönsted y Lowry definen los ácidos como las especies que tienen tendencia a donar protones y las bases como las especies capaces de aceptarlos. La neutralización según esta teoría es la transferencia de un protón de un ácido a una base en cualquier disolvente. La formación de sales también está induida dentro de los procesos ácido-base porque conlleva la transferencia de un protón desde un ácido a una base. Para Brönsted- Lowry ya no hay una dasificación absoluta de acidez, neutralidad o basicidad. Según esta teoría, la fuerza de un ácido se mide por la mayor 0 menor tendencia a donar un protón y la fuerza de una base por su mayor o menor tendencia a captarlo. Cuantitativamente se mide por el grado en que los reaccionantes se convierten en productos, pero el grado de esa reacción depende tanto de la tendencia de un ácido a ceder un protón como la de la base a aceptarlo, es decir, son medidas relativas y la única manera de comparar las fuerzas de dos ácidos es tomando como referencia una misma base, que será el agua para reacciones en disolución acuosa.

También en 1923, Lewis propone una teoría ayos postulados llevan a la condusión de que la neutralización consiste en la formación de un enlace coordinado entre la base que cede el par de electrones y el ácido que los acepta. Esta definición tampoco ofrece un criterio absoluto de dasificación de una sustancia como ácida, neutra o básica sino que siempre dependerá de la/s sustancia/s con las que se compare.

En 1939, Lux y Flood definieron como base toda sustancia capaz de ceder iones óxido y como ácido, las sustancias capaces de aceptarlos. Tanto la teoría de Brönsted-Lowy como la de LuxFlood son dos casos particulares de las teorías «ionotrópicas», es decir, definiciones de ácidos y bases basadas en los procesos de transferencia de iones, protones en el primer caso e iones óxido en el caso de Lux-Flood (Moeller, 1988).

En este mismo año, Usanovich (1939) define los ácidos como las sustancias que forman una sal con las bases por un proceso ácido-base, que da cationes o que se combina con los aniones o con los electrones. De forma similar, las bases son las sustancias que reaccionan con los ácidos, que dan aniones o electrones o que se combinan con los cationes. Hay que destacar que en esta teoría se induyen todos los procesos ácido-base posibles: transferencia de protones, de otros iones (los del disolvente, iones óxido, iones hidroxilo, etc.) o de electrones.

La revisión histónica que acabamos de presentar muestra las modificaciones que han sufrido las definiciones de ácido, base y neutralización según las diferentes teorías al uso. Una amplia revisión 
del desarrollo histórico que han sufrido los conceptos relacionados con los procesos ácido-base, en la que se induyen todas las teorías y los límites de aplicación, la ofrece Moeller (1988). El estudio realizado sobre los conceptos permitió entonces pensar en una propuesta para la enseñanza de la química, que se fundamentara en la construcción científica de los conceptos.

\section{Metodología}

Para la realización de la propuesta se partió de la idea de que un problema es una situación o cuestión, que se expone al estudiante con la intención de que este se interese por el tema y además lo lleve a generar nuevos conocimientos. De acuerdo García (2003), un problema se puede definir desde dos perspectivas una "desde el grado de dificultad que presente al individuo" y la otra "desde el camino utilizado para su solución". En el primer criterio se genera un problema cuando no existe respuesta alguna por parte del estudiante a la situación planteada, en este sentido se presenta como una novedad una o varias preguntas que "generan tensión en el pensamiento productivo de los individuos y cuya solución requiere de la búsqueda de nuevos conocimientos. Por otra parte un problema definido desde el camino definido desde el camino utilizado para su solución se puede considerar como una situación en la aul se requiere del individuo, un tratamiento distinto de una mera aplicación rutinaria de formulas de formulas. Interpretando lo expuesto por el autor, un problema puede ser una serie de situaciones que en un contexto específico generan en el estudiante un interés que lo motiva a buscar nuevos conocimientos que le permitan explicar la situación, lo que hace que se hagan nuevas elaboraciones, que se apartan del sentido común y que se sustentan en teorías y leyes que el estudiante incorpora en sus explicaciones para solucionar el problema.

Por lo que para la realización de este proceso se diseño una serie de actividades para evidenciar la apropiación de los conceptos y las ideas que poseían los alumnos de secundaria, por eso se realizó un instrumento de ideas previas en donde se encontraban diferentes tipos de preguntas, algunas de selección múltiple y otras donde el estudiante debía justificar su respuesta, con el fin de evidenciar si esos conocimientos o ideas altemativas que poseían, están o no guiados hacia lo que es el concepto como tal. A partir de ellos se realizó un análisis minucioso que nos permitió implementar las actividades propuestas y como dirigirlas a alcanzar el objetivo propuesto, por cada unas ellas para que los estudiantes apropien los conceptos trabajados. Para darle una validez a este test de ideas previas se hizo evaluación detenida por parte de diferentes docentes con especializaciones en la enseñanza de las ciencias, lo aral nos dará más confianza en los resultados obtenidos.

A partir de esto se realizaron un serie de actividades en donde se interaccionó trabajos de laboratorio, talleres de lecto-escritura, apoyo audiovisual y seminarios, pero todo encaminado a el diario vivir del estudiante, con el fin de contextualizar al estudiante ,se formuló una situación desde la acidez estomacal, para lo cual los reactivos utilizados fueron productos muy cotidianos y de fácil adquisición en el caso de las prácticas de laboratorio, para las lecturas se emplearon ejemplos de la vida real con lo es la acidez estomacal y por último se realizaban explicaciones para adarar las dudas que pudiera quedar después de la realización de cada una de las actividades, esto a partir de una retroalimentadión, lo que lleva a evidenciar las falencias o dificultades que pudieran presentar durante la actividad. Además de cada una de las ayudas mencionadas los estudiantes reciben un material físico para ellos que les sirve de apoyo durante el transcurso de la investigación.

\section{Resultados y discusión}

\section{Análisis conceptual}


Basados en los resultados se evidenció que los estudiantes mostraron una mejor apropiación de los conceptos, ya que cada una de las actividades las pudieron asociar con su diario vivir, al empezar la investigación el test de ideas previas nos mostro que los estudiantes tenían una vaga idea sobre los conceptos trabajados, a partir de la realización de cada una de las actividades se observó que los alumnos se identificaban más con la temática y había una apropiación mayor del concepto. Otro factor que influyo en este resultado fue la utilización de situaciones problema a partir de ejemplos de la vida cotidiana lo que ayuda a que ellos se interaccionen con el ejercicio y sea asociado a diversos hechos de su desarrollo personal.

Análisis Procedimental

El procedimiento que se llevó a cabo en la investigación fue el apropiado y arrojó resultados satisfactorios, debido a un análisis previo de la apropiación de los conceptos que tenían los estudiantes, por lo cual cada una de las actividades planteadas estaban encaminadas a reparar esos vacios conceptuales que tenían los alumnos sobre el tema, logrando que ellos al final del proceso presentaran un aprendizaje significativo del concepto y pudieran argumentar sus respuestas, lo que muestra que el proceso se llevó de una manera lógica y permitió alcanzar las metas propuestas.

\section{Análisis Actitudinal}

La actitud de los estudiantes frente al tema y cada una de las actividades fue muy favorable, sin embargo, el desarrollo del trabajo fue satisfactorio ya que la participación de los estudiantes fue activa y la relación que hubo entre docentes en formación y estudiantes fue favorable para el desarrollo de la investigación, esto se debió a que el distribuir en diferentes grupos los educandos ayudó a que el aprendizaje fuera más personalizado y la interacción que tenía el coordinador del grupo fuese más cercana a los alumnos, así el desarrollo de cada una de las actividades planteadas se hizo de una manera más directa con cada uno de los estudiantes y la comprensión de los conceptos a trabajar se facilitara y así poder hacer que el alumno adquiera ese conocimiento de una forma correcta y el afianzamiento al que deseábamos llegar con la unidad fuera satisfactorio tanto para los estudiantes como para nosotros como docente en formación.

\section{Conclusiones}

> La investigación se basó en la realización de una unidad didáctica guiada a partir de la resolución de problemas, ya que se toma un caso de la vida cotidiana para la enseñanza del concepto ácido-base, marcando gran diferencia frente a los ejercicios cotidianos trabajados normalmente en química, ya que para la resolución de la situación Problémica trabajada en ésta unidad didáctica, requiere que los estudiantes den explicaciones coherentes al conjunto de datos presentados en la situación relacionados al contexto en el que viven.

> Muchos de los estudiantes, no tenían preconceptos correctos acerca de lo que es un ácido, una base o neutralización, por lo cual fue crucial el buen manejo de las actividades propuestas en la unidad didáctica, ya que a partir del interés que los estudiantes prestarán, dependía también la comprensión de los conceptos por parte de ellos durante el desarrollo, comparándolo con la situación cotidiana (acidez estomacal).

- Es importante en una unidad didáctica, hacer uso de una terminología dara, lo menos técnica posible, además del uso de actividades adeauadas que permitan la enseñanza de un concepto con un fin satisfactorio y viable, y sobre todo, daro para los estudiantes.

D Es importante que un estudiante pueda argumentar lo que aprende, es decir, lleve a cabo una buena estructuración de los concepto que va adquiriendo, para evitar confusiones cognitivas 
con cada uno de los conceptos, sino que a partir de esto, haga diferencia entre cada uno de ellos comprendiendo el significado respectivamente.

$>$ Es fundamental escoger las actividades adecuadas para llamar la atención de los estudiantes, de forma que sean ellos mismo a través de la resolución de problemas, se adentren al concepto a manejar en la unidad con ayuda del docente.

> Por ultimo los docentes deben implementar una metodología que sea atractiva para los estudiantes no solo teniendo en cuenta la química como ciencia, sino todas las áreas, ya que esto va a favorecer el proceso enseñanza aprendizaje y el cambio conceptual va a ser mas fácil, al igual que llegar a identificar esos vacios conceptuales y poder atacar el problema de raíz, cabe resaltar que la utilización de las situaciones de la vida cotidiana de los estudiantes es primordial para alcanzar los objetivos propuestos, ya que esto le genera al alumno curiosidad hacia el tema y la apropiación de los conceptos se va a generar mas fácilmente y se llega a un aprendizaje autónomo por parte del estudiante.

\section{Bibliografía}

ARRENHIUS (1887), "On the Dissociation of Substances Dissolved in Water" Zeitschrift fur physikalische Chemie, I, 631

BRÖNSTED, J.N. (1923). Rev. Trav. Chim, 42, p. 718

FRANKLIN, E.C. (1912). Am Chem. J., 47, p. 285.

LOWRY, T.M. (1923). Chemi Ind. (Lond.), 42, p. 43

LUX, H. (1939) Z. Elektrochem, 45, p. 303

MOEUER, T. (1988). Química inorgánica. Barcelona: Reverté

USANOVICH, M. (1939). J. Gen. Chem. (USSR), 9, p. 182

GARCIA, J. J. (2003) Didáctica de las ciencias. Resolución de problemas y desarrollo de la creatividad. Colombia Cooperativa Editorial Magisterio. 